\title{
Editorial
}

Journal of Innate

Immunity

\section{Posttranslational Modifications in Innate Immunity}

\author{
Jan Potempa ${ }^{a, b}$ \\ ${ }^{a}$ Department of Microbiology, Faculty of Biochemistry, Biophysics and Biotechnology, Jagiellonian University, \\ Krakow, Poland; ' University of Louisville School of Dentistry, Oral Health and Systemic Disease, Louisville, Ky., USA
}

The term posttranslational modifications (PTMs) refers to a broad array of versatile chemical alterations of translated proteins. All of these modifications are enzyme catalyzed and allow for fast transformation of protein activity enabling a well-tuned regulation of cell function and response to environmental cues. In this way PTMs add a layer of complexity to gene-encoded protein function, both in prokaryotes and in eukaryotes. PTMs can be reversible or irreversible. Reversible modifications rely on specific enzymatic attachment of simple chemical groups including, for example, phosphate, acetate, methyl- or hydroxyl-groups, or complex molecules such as lipids, carbohydrates, ADP-ribose, or AMP.

Protein phosphorylation catalyzed by kinases, the enzymes transferring a phosphate group from ATP onto the hydroxyl group of serine, threonine, or tyrosine residues in proteins, is the most common PTM. This covalent modification of proteins, often occurring in a cascadelike manner, together with target proteins dephosphorylation by phosphatases, plays a fundamental role in the regulation of numerous signaling pathways in cells. Other common reversible PTMs include the covalent linkage of small proteins, such as ubiquitin and ubiquitin-like proteins (UBLs). On the other hand, enzymatic modification of amino acids, such as deamination of glutamate (transforms Glu into Gln) or deimination of arginine (converts Arg into citrulline), together with proteolytic processing of proteins, represent nonreversible PTMs.
Taking into account that a large number of nascent gene translation products are subject to limited proteolytic processing, proteolysis is one of the most common PTMs and due to its irreversible nature needs to be very precisely controlled on multiple levels.

Since PTMs play a key role in fundamental cellular processes, including the immune response, it is no surprise that these reactions are targets for virulence factors produced by viruses and pathogenic bacteria. By interfering with their host PTM pathways, pathogens disable or hijack for their own benefit host antibacterial mechanisms, manipulate inflammatory reactions, and build up an intracellular or extracellular niche for their survival and proliferation. Significantly, the host targets are mostly limited to several classes of molecules which play a fundamental role in endosome maturation, host cell cytoskeleton remodeling, and the host immune response [1]. In this context it is not surprising that regulatory GTPases are the most common target since they work as central molecular switches of eukaryotic cells regulating cytoskeleton function and membrane trafficking [2]. Alone, Rho and Rab GTPases are modified by adenylylation (AMPylation), ADP-ribosylation, N-acetylo-glucosamination, glucosylation, proteolysis, phosphocholination, transglutamination, deamination, and polyamination by virulence factors produced by a large array of bacterial pathogens $[1,2]$. A second most frequently manipulated PTM pathway is kinase signaling involved in

\section{KARGER}

Fax +4161306 1234

E-Mail karger@karger.ch

www.karger.com
(C) 2012 S. Karger AG, Basel

1662-811X/12/0042-0119/\$38.00/0

Accessible online at:

www.karger.com/jin
Dr. Jan Potempa

Department of Microbiology, Faculty of Biochemistry, Biophysics and Biotechnology Jagiellonian University

ul. Gronostajowa 7, PL-30387 Krakow (Poland)

Tel. +48 12664 6343, E-Mail jan.potempa@uj.edu.pl 
the function of the nuclear factor- $\kappa \mathrm{B}(\mathrm{NF}-\kappa \mathrm{B})$, mitogenactivated protein kinase (MAPK), phosphatidyl inositol 3-kinase (PI3K)/Akt, and p21-activated kinase (PAK) pathways [3]. This is in keeping with the pivotal role of these kinases in regulation of the innate and adoptive immune response.

In this issue of the Journal of Innate immunity, we focus on selected aspects of PTM hijacking by bacterial pathogens in the context of the effect on innate immunity. In the first review contributed by Garbe and Collin [4], an underappreciated side of PTMs, namely hydrolysis of host glycoprotein glycans by bacterial glycosidases, is presented. Based on available data and their own investigations, the authors discuss the ability of bacterial glycoside hydrolases to modulate glycosylated effector molecules of the immune response in such a way that this contributes to bacteria survival and proliferation. The conservation and abundance of putative hydrolase genes in the genomes of both bacterial pathogens and commensals argues that microorganisms commonly use this PTM strategy.

Several bacterial toxins including anthrax lethal factor, botulinum toxins, and tetanus toxin have turned out to be extremely specific proteases and are considered a leading example of proteolytic PTMs. The same is true with respect to the streptococcal IdeS protease, the action of which, though not as dramatic as that of the toxins, significantly contributes to the success of Streptococcus pyogenes as a versatile pathogen. In his review, von PawelRammingen [5] discusses unusual features of IdeS as the protease specific for the IgG hinge region, the role of the enzyme in S. pyogenes pathogenicity, and a possible therapeutic application of this bacterial protease.

Many bacterial pathogens developed strategies to usurp the host proteolytic activity to their advantage, and these can be considered specific cases of PTM. Activation of plasminogen by streptokinase and staphylokinase is an illustrious example due to application of the latter protein in thrombolytic therapy. Here, the ability of Staphylococcus aureus to modify the coagulation cascade to avoid opsonophagocytic clearance by host immune cells and disseminate via thrombolytic lesions using coagulase and von-Willebrand binding protein is the subject of the review presented by McAdow et al. [6].

Shen [7] summarizes the current knowledge on mechanisms by which glycosylating toxins (TcdA and TcdB) and a binary toxin (CDT) of Clostridium difficile disrupt target cell function. The function of the multidomain glycosylating toxins is presented in the context of protein structure, with the role of each domain illustrated in detail. This includes a fascinating description of the cysteine protease domain (CPD), which functions as a biosensor of the eukaryotic cell environment as it is allosterically activated to proteolytically release the $\mathrm{N}$-terminal glucosylating toxin domain by the cytosolic, eukaryotic-specific small molecule inositol hexakisphosphate $\left(\operatorname{InsP}_{6}\right)$. The $\mathrm{CPD}$ is found in several large multidomain glucosylating toxins and MARTX toxins [8], suggesting that bacteria commonly use this unique, double-PTM mechanism.

SUMOylation consists of the covalent conjugation to a target protein of an ubiquitin-like protein referred to as SUMO (small ubiquitin-like modifier). The addition of SUMO to target proteins requires a set of different enzymes in a manner similar to ubiquitylation. This reversible modification regulates such cellular processes as nuclear transport, transcription, chromosome segregation, and DNA repair. In addition, SUMO signaling is integrated with other signal transduction pathways [9], which apparently makes this pathway an attractive target for bacterial virulence factors. In the last review, Békés and Drag [10] focus on the most recently elucidated pathogen strategies exploiting the SUMO system of host eukaryotic cells.

\section{References}

1 Ribet D, Cossart P: Post-translational modifications of host cells during bacterial infection. FEBS Lett 2011;584:2748-2758.

$\checkmark 2$ Aktories K: Bacterial protein toxins that modify host regulatory GTPases. Nat Rev Microbiol 2011;9:487-498.

3 Krachler A, Woolery AR, Orth K: Manipulating of kinase signaling by bacterial pathogens. J Cell Biol 2011;195:1083-1092.

4 Garbe J, Collin M: Bacterial hydrolysis of host glycoproteins - powerful protein modification and efficient nutrient acquisition. J Innate Immun 2012;4:121-131.
5 von Pawel-Rammingen U: Streptococcal IdeS and its impact on immune response and inflammation. J Innate Immun 2012;4:132140.

6 McAdow M, Missiakas DM, Schneewind O: Staphylococcus aureus secretes coagulase and von Willebrand factor binding protein to modify the coagulation cascade and establish host infections. J Innate Immun 2012; 4:141-148.

7 Shen A: Clostridium difficile toxins: mediators of inflammation. J Innate Immun 2012; 4:149-158.
8 Egerer M, Satchell KJ: Inositol hexakisphosphate-induced autoprocessing of large bacterial protein toxins. PLoS Pathog 201; 6:e1000942.

$\checkmark 9$ Gareau JR, Lima CD: The SUMO pathway: emerging mechanisms that shape specificity, conjugation and recognition. Nat Rev Mol Cell Biol 2010;11:861-871.

10 Békés M, Drag M: Trojan horse strategies used by pathogens to influence the small ubiquitin-like modifier (SUMO) system of host eukaryotic cells. J Innate Immun 2012; 4:159-167. 